referred to unessential substances which are accidentally enclosed in the corpora amylacea.

Since then a positive amyloid reaction appears almost constantly, and since no proofs that the degeneration is of other than amyloid character are at hand, I believe I may conclude with Hildebrand, that the presence of amyloid matter in the concretions may be fairly assumed.

The origin of the corpora from rings of epithelium explains clearly enough their concentric arrangement. This explanation applies, however, to those concretions only, showing broad lamince and giving the iodin reaction.

The origin of those corpora which have sharply marked concentric lines and which do not react with iodin and sulphuric acid, can not be explained in this way. These bodies have exactly the same appearance as the unstainable yellow rings in the otherwise stained concretions which are described above. They have the usual color of the prostatic contents. These are the bodies which, according to Seigert, are the product of hyaline degeneration of epithelium. The Innermost layer of these corpora were very often of triangular form. Round about this layer were angular layers; the angles becoming more obtuse, and the number of the same increasing toward the periphery, the shape became gradually circular. It was remarked that the sides of the triangular layer were made up of spindle-shaped, colorless formations.

As is well known, such spindle-shaped formations are abundant in the prostatic juice. Occurring in the prostate they are called Boettcher's crystals. They are identical with those formations which in the lungs and central nervous system are known as CharcotLeyden crystals.

North Delaware St.

REFERENCES.

1. Virchow: Virchow's Archiv, Vols, jand 6.

Virchow: Cellular Pathologie, Fourth Edition.

Friedrich: Virchow's Archiv, Vols. 9

Cornil: Arch. de Phys. norm. et Path., 1875.

Zahn: Virchow's Archiv, Vol. 72.

Favre: Thise de reneve, 1879

Stilling; Virchow's Archiv, Vol $9 \mathrm{~s}^{\circ}$

Furbringer: Zeitschrift für klin. Med., Vol. :;

Posner: Zeitsehrift fïr Afed. Vol, 16

Posner: Virchow's Archiv, vol. 79 .

Hoppe-seyler: Physiol. Chemit. Isis.

o. Israel: Practicum.

Seigert: Virchow's Archiv, vol. 129

. II ildebrand: Virchow's Archiv, Vol. 140.

Langhans: Virchow's Archiv, Vol. 120.

Jürgens: Virchow's Archiv, Vol. 6.).

1. Lubarsch: Virchow's A rchiv, Vol. 13.

19. Sir Henry Thompson: Dis, of the Prostate, London, Ixri:

20. Ebstein: Zeitschrift für klin. Med., Vol. 40

21. Rindfleisch; Path. Gewebelehre, Fourth Edition.

22. Cohn: Fortschritte der Med., Vol. 14, No. 16.

23. Von Recklinghausen: Virchow's Archiv, Vol. s4.

24. Czerny: Archiv. für experim. Path. u. Pharmakol., Vol. 31.

Furhringer: Berliner klin. Wochenschrift, 1\$85, No. 49

Haussmann: Berliner klin. Woehensehrift, 1893, No. 28

27. Erhlich: Zeitschrift für klin. Med., Yol. 6.

\section{THE NATURE AND VARIETIES OF ANEMIA.}

Read in the Section on Practice of Medicine, at the Forty-eighth Annual Ieeting of the American aredical Association held at

Philadelphia, Pa., June 1.4, $189 \bar{i}$.

BY ALFRED STENGEL, M.D.

Assistant Director Pepper Laboratorv of Clinical Medicine: Assistant Physician to the Hospital and Instructor in Clinical Medicine, PHILADELPHIA, PA.

It would be hopeless to attempt to review, even imperfectly, all of the questions of importance connected with the subject of anemia in the time at my disposal; but I believe it wisest to consider a few of the problems relating to the general conception of the nature of this disease or group of diseases, and to dis- cuss briefly the varieties generally admitted to our nosology.

It is difficult to define anemia in a way satisfactory to the modern pathologic requirements. The older authors simplified the matter in conformity with the scope of their pathology, by defining it as bloodlessness, and by associating the clinical appearance of pallor with the disease anemia. Later, after the chemical studies of Andral and Gaveret. Becquerel and Rodier and Schmidt, names which must forever occupy a conspicuous place in the history of hematology, clearer conceptions of the exact nature of the disease seemed within reach, though time and repeated studies along the lines established by these pioneers have tailed to realize the hope entertained in the beginning. The general revolution in pathology, stimulated by the genius of Rokitansky and especially by Virchow; and the special studies of the blood by Virchow, Bennett, Cohnheim and Neumann gave the study of the blood a different direction, bringing morphology into greater prominence and leading to various theories regarding the formation of the blood and the nature of anemia. Meantime methods of enumerating the cellular constituents and determining the amount of hemoglobin were introduced and perfected, and the morphological studies were brought to their present state through the labors of Bizozzoro and Hayem, and particularly of Ehrlich. During the past ten or fifteen years the influence of the last-named authority has been universally felt, and his opinions, based upon more accurate methods established by himself and his pupils, have everywhere dominated. So powerful has been this mastery that in certain quarters morphology alone has been considered the test by which anemic conditions should be judged of, and by which contending theories regarding the essential nature of anemia should be harmonized or rejected. Every active movement in science or politics or art carries its adherents beyond the proper limits and so it has been in hematology. Powerful for good, as has been the teaching of Ehrlich in renewing interest in the study of the blood and in the direct results, it merits the reproach of narrowing for a time the horizon of hematology in limiting attention to the corpuscles and indirectly discouraging chemical and physiologic studies of a broader kind. The return movement has happily set in, with what useful result remains to be determined.

A primal consideration in the study of the blood is the proper conception of its relations to the general organism and its limitations as a distinct issue. Virchow described it as a liquid tissue having close analogies with the solids. Standing alone this dictum must lead to erroneous notions regarding anemia. In every sense the blood is a dependent fluid. drawing no doubtfrom all the structures of the body in varying degree some part of the elements that make for preservation of its integrity. In how far the so-called blood-making organs are operative in predominance over the other structures, can not as yet be shown. Reasoning from the basis of morphology the spleen, the marrow and the lymphatic tissues are preëminently essential structures, and with a view of the analogies furnished by recent studies of the internal secretions as a whole, it would seem likely that these structures have other, and perhaps more important, functions than the mere manufacture of corpuscles. The therapeutic use of organic extracts, and particularly of those derived from bone-marrow, $t \curvearrowright m p t s$ me to a slight 
diversion. Individual experience varies widely with regard to the efficiency of these and my own has been unsatisfactory. If, however, further trials should lead to uniform and positive results, we must regard this as a proof of secretory function rather than of the usefulness of marrow as a hematinic food. The amount of iron contained is no greater than that in other foods, and the form of its combination does not differ essentially from that in other animal diet. For the present, however, the relations of the bone-marrow in the direction of internal secretion are undetermined, and can not be utilized for the purpose of substantiation or disproof of any theory.

The individual life of the red blood corpuscle is a limited one, though we can not as yet assign a definite duration. Its birth and its ultimate dissolution are obscure. Certain it is, however, that both processes are dependent largely or wholly on the integrity of the other tissues and organs, and the opposing views of Virchow, Neumann and others, and of Biesiadecki and Löwit regarding the secondary or primary nature of leukemia (in the sense that this disease is in essence a disease primarily of the blood-forming organs, or on the other hand of the blood itself), might almost be decided in favor of the former on this consideration alone. Morphologic studies, as those of Biesiadecki in particular, led to the conception that leukemia is an essential disorder of the blood, characterized by a retardation of the evolution of the leucocytes, and the study of morphology alone would warrant further adherence to this view. The wider pathologic studies of Flemming, Neumann and Virchow and particularly the chemical experiments of Horbacewski and others, abundantly demonstrated the narrowness of the older conception and substantiate for this disease, what may be asserted of anemiæ in general, that it is altogether secondary to primary organic disorders.

Let me take another point of view and consider for a moment the subject of post-hemorrhagic anemia. This is the purest and most direct variety of which we have knowledge. Animals will tolerate great loss of blood and may thus be made intensely anemic. The effect of the hemorrhage is not a mere reduction of the quantity of the blood but a rapid deterioration of quality in certain directions. This is well known and is easily demonstrated in man as well as in experimental work. The primary result of the hemorrhage is a reduction in the quantity of the blood. Physiologists have shown that serious results in the way of ischemia, or local anemia, are prevented by the coincident and proportionate vasomotor contraction, the blood pressure remaining approximately normal. Later the blood becomes more fluid and the number of corpuscles together with the proportion of the hemoglobin falls. Liquid is in some way absorbed by the blood. and the ready explanation that the vossels are filled by the juices of the various tissues is at hand. There is not, however, a mere dilution with water as chemical analyses readily demonstrate. For example I would quote the following experiment:

A small dog weighing sixteen kilos. was bled from the fugular vein one-third of his total blood (estimating the total quantity at one-thirteenth of the body weight) being removed. Before the experiment the following values were determined: Red blood corpuscles, $6,900,000$; white blood corpuscles, 42,000 specific gravity of blood, 1,059 ; specific gravity of serum, 1022.8 dry residue of blood, 23.2 per cent. ; dry residue of serum, 7 . per cent. Ten minutes after the bleeding small quantities were taken for examination and the following results obtained: Red blood corpuscles, $6,610,000$; white blood corpuscles, 19,600 specific gravity of blond, 1055.9 ; specific gravity of serum 1022.6 ; dry residue of blood, 21.6 per cent. ; dry residue of serum, 6.9 per cent.

It will be noted that there was no immedicte change in the quality of the blood; at least no striking change. Seven hours after the bleeding the following figures were obtained: Red blood corpuscles, 2,615,000; white blood corpuscles, 20,600.

Chemical analyses were not made at this time, but were determined at the next examination, forty-eight hours after the original bleeding. The animal was again etherized and blood removed from the femoral vein: Red blood corpuscles, $3,100,000$; white blood corpuscles, 26,000 ; specific gravity of blood, 1046.7 : specific gravity of serum, 1022.1 ; dry residue of blood, 10.47 per cent. ; dry residue of serum, 7.4.

From the similarity in the blood count at this and the previous examination, I assume that the specific gravity and the residue of the blood and serum would have been found practically the same at the previous examination. It is evident then that the mass of the blood is soon diluted after hemorrhage and the diluted fluid is approximately the same in density and probably in churacter as the original plasma. This is shown by the preservation of the normal conditions of the serum while the total blood has undergone diminution in weight and in solid residue.

The manner in which this change occurs is of very great physiologic interest and bears important relations to the etiology of anemia. Either there is a direct transfer of the liquids of the body to the vascular system through the walls of the vessels, and a depletion therefore of the general system in favor of the blood, or there are active secretory processes affecting the vessels, according to the theory of Heidenhain. It will be recalled that this observer found certain substances capable of stimulating active flow of lymph, the latter also being more concentrated than usual and the increased flow continuing for a considerable period. There is no increase of the arterial pressure. His own theory regarding the action of such substances is that there is a specific stimulation of the endothelial cells of the capillaries of a secretory kind. While physiologists in general have not supported Heidenhain uniformly, certain of his experiments raise questions that have not as yet been satisfactorily explained by any other hypothesis. For example, the presence of sugar or salts in the lymph of the thoracic duct in greater quantities than in the blood, is a fact that can not readily be explained on the theory of simple osmosis. For the present we can not do more than refer to this interesting physio. logic question, and cite it as having a bearing upon the general subject of the relations of the blood to organic metabolism, and the probable activity as secretory structures of tissues hitherto unassociated with such function.

In studying the relations of the corpuscles and plasma, the specific gravity of the blood and serum and the solid residues of these in cases of anemia, either primary or secondary, there is a striking resemblance to the conditions observed after hemorrhage The specific gravity of the blood falls in proportion to the reduction of coloring matter; the quantity of serum rises while its specific gravity and its solid residue alters little in comparison with the change in the blood as a whole. There is undoubtedly a transfer of fluid from the tissues to the blood stream. Older observers sought to explain the changes in density and constitution of the blood upon the assumption that water is added to the blood and albuminous elements abstracted, and this view has even recently 
found expression. The disproof is not far to seek. blood a normal condition was found. Of the same The studies of Grawitz have shown, as have recent sort is the pallor developed in visitors to tropical counexperiments of my colleague Dr. Taylor, that though tries. These cases of spurious anemia are doubtless the proportion of albumin in aliquot parts of serum dependent upon vasomotor changes rather than is reduced in severe anemias, the total quantity (tak- changes in the blood itself. They are of interest in ing into consideration the increased bulk of serum in connection with my present subject only from the the blood) is not reduced below the normal; indeed fact that they evidence the general relations of the it sometimes increases.

Let me next refer to the character of the red corpuscles in anemia. I need not detail the various changes of form and general structure, but will only refer to the differences in size, in shape, and to the alterations in internal structure (vacuolation and pseudo-vacuolation, altered color-reactions and the like). These are conditions well known in anemia and more or less proportionate to its degree. They find their counterpart, however, in the artificial anemia following hemorrhage, and here again in proportion to the grade of anemia. Imperfect hemogenesis (the term being restricted to the making of red blood corpuscles) and active hemolysis in this or that locality, have been too readily assumed in explanation of these conditions according to the individual views of various authors; and the possibility of these changes being dependent upon primary change in the general construction of the blood itself, has been too often disregarded. It is true that recent studies of the effects of the various hemolytic agents have tended to alter the direction of opinion, and I may cite the experiments of Silbermann, Heintz and Hunter, from many contributed in recent years. The thought, however, that in the spontaneous anemiæ, changes of the fluid of the blood account for alterations in the corpuscles, has not frequently found expression. Maragliano and Castellino and Gilbert, have made the more important of the scanty observations in this direction, studying the behavior of corpuscles when exposed to the action of serum of anemic diseases. These studies, though open to criticism for their crudeness, in a measure contribute support to the belief that the serum is largely responsible for many of the changes observed in the corpuscles. In my own experiments I have found the serum of cases of pernicious anemia, chlorosis and leukemia powerfully destructive to normal corpuscles; but I have as yet had no opportunity of investigating the serum after serious hemorrhages.

The number and bulk of the red corpuscles in anemia offer interesting problems for solution; but first I wish to refer, for a moment, to the relation of pallor to anemia. The appearance of the patient has always furnished the test by which the probable presence of anemia has been obtained, and formerly was the only test. It must be remembered, however, that pallid features do not of necessity proclaim an actual state of anemia. Some individuals are naturally pale as others are florid, and the condition of the blood is not of necessity altered from the normal in either case. Clinical studies of chlorosis and other anemic diseases further give evidence of the fact that, pallor of the skin or of the mucous membranes is not a reliable guide to the condition of the blood. It is well to bear in mind in this connection, that the pseudo-anemic condition may be rather suddenly developed and may persist for a greater or less length of time. Interesting cases of this description have been reported; among others one that I now recall in which a young woman, who had fallen and suffered great fright as well as some concussion, became excessively pale, suggesting the existvasomotor system to the distribution of blood.

Actual changes in the blood may occur in consequence of circulatory disturbances in persons residing at a considerable elevation, in those suffering with failure of the cardiac compensation and in consequence of peripheral congestion. Beginning with the last, I have frequently demonstrated the greater corpuscular richness of the blood of dependent parts over that of the finger held in the air. The difference has been variously estimated by investigators at from 10 to 15 per cent. In my own experiments the average has been about 8 per cent. In cardiac diseases there may be similar concentration of the blood and excess of corpuscles in the given quantity. In high altitude this is so regularly the case that this condition may be taken as one of the possible alterations effected by elevation that influence general organic process. We may deduce from these facts the general cenclusion that alterations in the number of corpuscles are of less importance than has often been maintained; and that the changes in the blood, in the direction of concentration, are probably the result of the disposition and distribution of the serum. I am aware that there are facts favoring the view that actual increased formation of corpuscles occurs in residents of high altitudes, but taken in connection with the rapidly induced changes in the number of corpuscles in congestion and similar conditions, the conclusion seems not unwarranted that the altered distribution of serum is the more important factor.

I can not now go into the matter of altered chemical relations of the blood in anemia; into the change of proportion of the different albuminous constituents and of the inorganic elements, of the presence of compounds usually found in but small proportions or not at all; but can only cite these in evidence of the riew that serum changes are of great if not commanding importance.

The estimations of the isotonic coefficients of the corpuscles and of the serum are of interest, though they have not as yet furnished definite results. There is a tendency, it may be asserted, toward decreased resistive power of the corpuscles in the anemix and perhaps a greater alteration of the hypertonic strength of the serum, the result of which is a lessened state of stability of the blood mass as a whole in anemia.

I have thus hastily sketched a few of the facts regarding anemia that are still most obscure, with the purpose of emphasizing this one point, viz: that it would be an error to consider the blood from the standpoint of the corpusclar elements alone. Perhaps it needed no extended argument to make this fact apparent, but in actual practical study the profession has been so prone to regard the corpuscular elements as the criterion by which to estimate anemia, that an elaborate consideration (not to speak of the sketchy discussion I have undertaken), would be justified. I am convinced that the more important factor in anemia is the altered character of the fluid, and that studies to be profitable must be directed hereafter to the determination of the relations of this to the genence of severe anemia; but on examination of the eral organic life; and that less attention may well be 
directed to the formation, number, or even the general characteristics of the corpuscles, though I would not for a moment underrate the value of these observations. We see, in every anemia, results of changes in the constitution of the fluid of the blood, and we see but few effects of altered corpuscular elements. The older authors believed that the reduced number of corpuscles and the reduced quantity of hemoglobin must interfere greatly with respiratory exchange of gases; but the accurate physiologic studies of recent years have shown that this is not the case. Indeed, there is if anything an increased respiratory exchange in anemia. Toxic elements undoubtedly occur in the plasma in these diseases though their nature is obscure. On this assumption we must explain the occurrence of degenerative changes in the solid tissues, notably in the heart, met with in pernicious anemia, and to a less extent in other anemic affections.

In considering the possibility of a comprehensive definition of anemia in the light of these considerations it is well to observe in the first place that no single constituent occupies a principal place. It is true that all the anemias of which we have knowledge are diseases in which there is reduction either in the number of corpuscles in the proportion of hemoglobin or both; but it does not follow that these are the essential conditions. Indeed, it is likely that these are but the more evident expressions of the altered general condition of the blood, other changes being obscure. A recent writer (Frederick Taylor) in discussing anemia in its various bearings explicitly states that the number and condition of the leucocytes may be disregarded and I cite this merely in evidence of the too narrow view commonly taken of the disease. I believe anemia to be a condition of deterioration of the blood with altered relations of the fluid and solid parts. In some cases the number of corpuscles may be approximately normal, the quantity of solid residue, however, being reduced. The change in such cases may affect the plasma primarily or the chemic construction of the individual corpuscle. The definition does not include purely quantitative anemia and for the definite reason that $I$ believe the condition of the blood is invariably altered in cases in which there is quantitative reduction. I can not now discuss the possibility of the existence of a continuous insufficiency of the quantity of the blood, but only state my belief in its occurrence.

The classification of anemias is extremely difficult. For clinical purposes we may still retain the terms primary and secondary, with the restriction, however, that these terms are used in a sense different from that originally intended. By the primary or essential anemia we may designate the group of diseases in which the more striking symptoms are those affecting the blood itself, or the blood-making organs with the blood. By a secondary anemia we would designate cases in which the condition of the blood is of less prominence than the primary disease which has brought with it a deterioration of the blood. Viewed from the standpoint of our modern pathology there is little doubt but that pernicious anemia, for example, is frequently a secondary anemia, using this term in its older sense; that is to say, pernicious anemia results from blood destruction dependent on organic disease or intoxication from some part of the organism and is not a disease dependent primarily upon disorder of the hematopoietic organs. It is true, however, that the primitive cause is always less conspicuous in this disease than anemia itself, whereas in the secondary anemias, in the clinical sense, the original disease is always prominent.

Chlorosis.-Chlorosis is undoubtedly a hemogenic disease, dependent on some defect in the general organism, affecting the blood-making power. It is well to recognize in the beginning that this disease can not be distinguished by the character of the blood alone. It is true that severe cases present a picture of the blood rarely seen in any other condition, but the absolute diagnosis can not thus be made. The strict morphologist and those who rely greatly upon clinical examination of the blood often state the contrary, but withont sufficient authority. The chloro-anemia of tuberculosis and syphilis and gastro-intestinal disease in some cases produces exactly or almost exactly the condition of the blood observed in chlorosis. This leads me to question the existence of chlorosis in the male. There is no a priori reason for disbelieving the occurrence of this disease in the male, but all clinicians are careful in their statements regarding this possibility and many frankly state that the disease occurs only in girls and women. In examining the reports of those who claim its existence in the male sex I have been struck by the imperfect manner in which the diagnosis has been established. A recent report offers no convincing evidence of any kind and seems to be based entirely upon the existence of a moderate oligochromemia. Certain competent authors however have claimed to have seen genuine cases in males, and I believe that one instance has come under my own observation. In this case the most searching examination failed to disclose any primary disease. The patient was pallid with a peculiar yellowish cast; there was no emaciation and the tissues were rather flabby. The patient was effeminate, poorly developed and hysteric. Palpitation and dyspnea occurred and there were murmurs over the heart. The number of corpuscles was almost normal, the hemoglobin was reduced to about 40 per cent.; constipation was marked. I can not now enter into a discussion of theories regarding the nature of the disease. The opening sentence of this section states my belief regarding the nature of the disease. I must, however, make allusion to one consideration, viz., as to the advisability of increasing the scope of the term chlorosis so as to include all anemic conditions in which there is excessive oligochromemia. My experience makes the decision easy. Clinical observation leaves no doubt as to the existence of a distinct morbid entity, that we term chlorosis. Reduction of hemoglobin without commensurate reduction of the corpuscles may be important in this affection, but it does not alone constitute the disease. What other alterations the blood may present of an essential kind has not as yet been determined. Undoubtedly, however, there is more than a mere change in the character of the red cells. Pernicious anemia.-The discussion of pernicious anemia need not detain us long. The evidences in favor of an hemolytic origin of this disease are well nigh absolute. I wish to consider only one question, viz., the scope of the term pernicious anemia and its limitations. Certain authors, notably Eichhorst, designate two varieties; the primary and the secondary, the latter term including the cases in which definite lesions, probably of etiologic significance, are found. For my own part I can see no justification of this course beyond the deference it implies toward traditional teaching. The first observers regarded pernicious anemia as essential or idiopathic, admitting 
no other variety. It is, however, undoubtedly true that many cases occur in which the clinical features are the same, the course and prognosis identical, but in which lesions presumably connected with the development of the disease are discovered. In these cases the evidences of hemolysis may be pronounced and the connection between the organic lesions and the anemia seems direct. On the other hand, the cases in which no such lesions are discovered may present evidences of hemolysis as clearly, as those with lesions and as the course of the disease is alike I would put all cases in the same group. The diagnosis of the disease therefore rests upon the condition of the blood and the general course of the affection, rather than upon any evidence or lack of evidence of apparent cause. Besides the classical cases of pernicious anemia I have observed one or two in which the appearances were somewhat different and which I believe may be of slightly different kind in nature. One of these cases affected a man past middle life who began to grow anemic without apparent cause and continued to grow more and more pallid until his death. The color of the skin was almost an alabaster white, there was decided emaciation, rarely if ever any fever, the urine was pale, non-albuminous and watery; the blood presented marked but not excessive oligocythemia $(2,000,000$ to $2,500,000)$, the hemoglobin was rather more decidedly reduced ( 30 to 35 per cent.). The corpuscles were altered in form and there were a few nucleated forms. The patient was exceedingly weak and in spite of active medication died after an illness of several months. A complete autopsy, including every structure of the body, was performed and no lesions were found excepting fatty degeneration of the heart, liver and kidneys, and edema of the lungs. There was no excess of iron pigment in the liver or spleen; the bone marrow was normal. I cite this case merely as an instance of obscure pernicious anemia, difficult to classify and perhaps representing a form of progressive fatal anemia of a kind different from ordinary pernicious anemia. The condition of the heart did not at any time cause prominent symptoms. I believe it was secondary.

Leukemia.-I shall say but a word or two regarding this. Its close relations with Hodgkin's disease is evidenced by the occasional transformation of the lat. ter into the former. Some have held that Hodgkin's disease represents an aleukemic stage of leukemia. This may be true of some instances and I am confident the one disease may lead directly to the other; it does not follow, however, that such a tendency exists in all cases. Regarding the nature of these diseases I can not but consider them as closely associated with sarcoma

Infantile pseulo-leukemia.-Von Jaksch described a form of disease, in 1889 , under this heading and held it to be a distinct variety. Similar conditions had been previously more or less satisfactorily described by the Italians. A careful review of the literature of this disease shows that it possesses no distinctive features to warrant its classification as a distinct entity. A large number of the cases are undoubtedly closely associated with rickets; in others syphilis may be the important factor. The pathologic lesions are those of chronic hyperplastic disease of the spleen and liver and do not warrant the assumption that there is a separate pathology. The marked leukocytosis is striking but does not constitute a feature of sufficient stability to separate these cases from other varieties of splenic enlargement.

Splenic anemia.-This term has been variously used. There are undoubtedly cases of Hodgkin's disease in which the spleen enlarges markedly while the lymphatic glands remain comparatively little affected. Moreover there are cases of secondary splenic hypertrophy in the course of chronic malaria, rickets, syphilis and other diseases in which the anemia is pronounced and the original etiologic factor is difficult to determine. Cases that have come to autopsy, or in which the character of the spleen could be determined at operation for splenectomy, showed that the condition of the organ is for the most part that of chronic connective tissue hyperplasia with some true splenic hypertrophy. It is true there are some cases recorded in which the appearances were those met with in Hodgkin's disease and leukemia, but such have been very unusual. These observations warrant our disregarding entirely the term splenic anemia. I do not know of the existence of a separate disease that we may dignify with this title, or with such names as "primary splenomegaly" or "idiopathic enlargement of the spleen." The conditions of the blood as the characteristics of the spleen do not differ from those met with in well-marked secondary anemia or splenic enlargements.

\section{ON THE STUDY OF THE BLOOD.}

Presented in the Section on Practice of Medicine, at the Forty-eighth Aunual Meeting of the American Medical Association, at Phỉladelphia, Pa., June 1-4, 1897.

\section{BY A. E. TAYLOR, M.D.}

A SNOCIATE IN CIINICAL MEDICINE, PEPPER LABORATORY OF CLINICAI MEDICINE, UNIVERSITY OF PENNSYLVANIA.

PHILA DELPHIA, PA.

The present tendency in the study of the blood is one directed by almost boundless enthusiasm. The diagnostic success of blood examinations for the protozoa of malaria, the differentiation of diseases with leucocytosis from those without it, the separation of the essential anemiæ, the serum diagnosis of typhoid fever and the frequent positive results of blood cultures, have been of such striking practical and pathologic importance that confidence in the results of blood study is wide-spread and profound. Now, therefore, is the proper time for care and caution. It is clear that many of our present methods are inadequate and that their results are being accepted for more than their real value. For the further successful study of the blood not only must it be studied in its widest biologic relations, but the methods and technique must be greatly enlarged and made more accurate and likewise more simple. At the beginning I wish to recall your closer attention to venepuncture as a most suitable method of obtaining blood for study. The blood drawn from the puncture in the finger or ear can be used only for the count, the hemoglobin estimation, and for morphologic study; for other methods where more blood is required venous blood must be used. To do venepuncture a bandage is applied above the elbow or ankle, the skin antiseptically cleansed, a spot over a good-sized vein anesthetized with ethyl chlorid and a large aseptic hypodermic needle, such as is commonly used in surgical work, inserted into the vein. It is very easy to insert such a needle into the distended vein, the patient feels no pain, the operation can be so conducted that the patient sees no blood, and after the withdrawal of the needle only a pin-point mark remains, which causes no inconvenience. From such a puncture several fluid 